\title{
EFFICIENT ENERGY SAVING AND DATA COLLECTION IN WIRELESS SENSOR NETWORKS
}

\author{
R. Evangelin Hema Mariya. \\ Kingston Engineering College, \\ Vellore-59, India
}

\begin{abstract}
Energy efficiency operations are essential in increasing the lifetime of Wireless Sensor Networks. Clustering sensor nodes is an energy saving based interesting technique for achieving these goals. The main challenge in wireless sensor network is to optimize energy consumption when collecting data from sensor nodes. Efficiently collecting the data in wireless sensor networks plays a key role in power conservation. In this paper, a new energy-efficient approach for clustering nodes in adhoc sensor networks is proposed to collect data from sensor nodes and to reduce energy consumption using modified tabu search method, which enables with low communication cost. Dynamic Tabu search uses a local or neighborhood search procedure to iteratively move from a solution $\mathbf{X}$ to a solution $\mathbf{X}^{\prime}$ in the neighborhood of $\mathbf{X}$, until some stopping criterion has been satisfied. Communication between the distributed cluster heads is to be achieved by low cost. This approach is suitable to avoid the energy wastage during the transmission and to prolong the lifetime of sensor networks. The MNL (Maximising Network Lifetime) algorithm is being added to prolong the life time of the distributed network. NS-2 is used to perform the progress. The performance of distributed approach is to be compared with the centralized clustering approach using Dynamic modified tabu search.
\end{abstract}

Keywords: wireless sensor networks, clustering, efficient energy saving, Distributed cluster heads, maximize network lifetime.

\section{INTRODUCTION}

Sensor networks have recently emerged as an important computing platform. Sensor nodes are typically less mobile and more densely deployed than mobile ad-hoc networks (MANETs).The wireless sensor network (WSN) technology is a key component for ubiquitous computing. A WSN consists of a large number of sensor nodes. Each sensor node senses environmental conditions such as temperature, pressure and light and sends the sensed data to a base station
(BS), which is a long way off in general. Since the sensor nodes are powered by limited power batteries, in order to prolong the life time of the network, low energy consumption is important for sensor nodes. In order to reduce the energy consumption, a clustering and data aggregation approach is being used. In this approach, sensor nodes are divided into clusters, and for each cluster, one representative node, which called cluster head $(\mathrm{CH})$, aggregates all the data within 
the cluster and sends the data to BS. Since only $\mathrm{CH}$ nodes need long distance transmission, the other nodes save the energy consumption and increase the scalability and lifetime of the network.

Clustering is one of the fundamental issues in wireless adhoc and sensor networks. In clustered sensor networks, clusterheads $(\mathrm{CH})$ are responsible for data fusion within each cluster and transmit the aggregated data to the remote Base station (BS). With clustering the network payload has been greatly reduced i.e. battery energy can be considerably saved. In order to prolong the network lifetime, energy-efficient protocols should be designed for the characteristic of WSN. Efficiently organizing sensor nodes into clusters is useful in reducing energy consumption. Many energy-efficient routing protocols are designed based on the clustering structure. The clustering technique can also used to perform data aggregation, which combines the data from source nodes into a small set of meaningful information. Under the condition of achieving sufficient data rate specified by applications, the fewer messages are transmitted, the more energy is saved. Localized algorithms can efficiently operate within clusters and need not to wait for control messages propagating across the whole network. Therefore localized algorithms bring better scalability to large networks than centralized algorithms, which are executed in global structure. Clustering technique can be extremely effective in broadcast and data query. Cluster-heads will help to broadcast messages and collect interested data within their own clusters. During data collection, two mechanisms are used to reduce energy consumption: message aggregation and filtering of redundant data. These mechanisms generally use clustering methods in order to coordinate aggregation and filtering..The essential operation in sensor node clustering is to select a set of cluster heads among the nodes in the network, and cluster the rest of the nodes with these heads. This paper proposes a Distributed clustering mechanism equipped with energy maps and constrained by Quality-ofService (QoS) requirements. Such a clustering mechanism is used to collect data in sensor networks.

\section{PROBLEM STATEMENT}

The Central approach is less efficient than the distributed approach in the cluster building phase. The nodes in the centralized approach have to send their information to a central node that collects all of the information and runs the algorithm to build the clusters. Energy consumed by building cluster and the energy consumed during the data collection phase is more in centralized approach.

\section{RELATED WORK}

There is a large body of related work in cluster formation and the communication between them that attempts to solve similar problems using various techniques. Moussaoui et al [1] discusses a "novel energy efficient and reliable clustering (EERC) algorithm" rebuild the clusters when there is a heavy load in the $\mathrm{CH}$. Furthermore, they have a great problem in reliability that cluster-heads are easy to be attacked. It may lead uselessness of the whole cluster, thus greatly reduce the network reliability.

Raghuwanshi et al[2] similarly use the cluster communication .Communication within the cluster takes place over one-hop distance while traffic moves through the network over multi-hops to points that are connected to a much larger infrastructure. A handshake takes place between the broadcasting cluster-head and the non-cluster-head neighbors, before any data transmission can begin. Each time the nodes in the network configure - new/mobile/hibernating nodes get discovered by the local search performed as a part of the dynamic clustering scheme. Nodes that are closer in distance can have lower energy levels than farther nodes and run out of battery power quickly. The broadcast is done to make the presence known to all neighbours at single-hop distance. Based on the assumption that at least one node is awake at one-hop distance, the corresponding cluster-head sets a timer for which it decides to stay as the cluster-head 


\section{International Journal of Computer Applications Technology and Research}

\section{Volume 1- Issue 3, 82-88, 2012}

Younis et al [3] proposes another clustering method for clusters in distributed manner. Network lifetime can be defined as the time elapsed until the first node (or the last node) in the network depletes its energy (dies).energy efficient clustering method is implemented using a protocol, HEED (Hybrid Energy-Efficient Distributed clustering), that periodically selects cluster heads according to a hybrid of their residual energy and a secondary parameter, such as node proximity to its neighbors or node degree. Cluster heads are randomly selected based on their residual energy, and nodes join clusters such that communication cost is minimized. Simulation results show that HEED prolongs network lifetime, and the clusters it produces exhibit several appealing characteristics.

Suchismita Chinara et al [4] propose a cluster head selection criteria using an adaptive algorithm. As the selected cluster heads form the routing backbone of the dynamic network, better stability is ensured by preferring low mobile nodes to act as cluster heads. The algorithm weight based distributed mobility adaptive algorithm DMAC aims to distribute the time for which a node is selected as cluster head in an uniform manner so that every node obtains nearly equal opportunity to act as a central router for its neighbor nodes .

El Rhazi et al [5] propose a data collection algorithm using energy maps. Data aggregation and filtering methods, which minimize transmitted messages over a network, are widely used at the moment to reduce power consumption.A new data collection mechanism that uses a distributed clustering method. The new cluster building approach is based on the network energy map and the QoS requirements specified by an application. The energy consumption model determines the sensor lifetime. The energy map, the component that contains information concerning the remaining available energy in all network areas, can be used to prolong the network lifetime. A novel data collection approach for sensor networks that use energy maps to reduce power consumption and increase network coverage is used. The nodes consume more energy compared to TAG.

Heinzelman et al [6] focus on the limits of the scalability of the protocol. For this, LEACH, application-specific protocol architecture is being proposed. LEACH, a protocol architecture where computation is performed locally to reduce the amount of transmitted data, network configuration and operation is done using local control, and media access control (MAC) and routing protocols enable low-energy networking. The advantage of rotating the cluster head position among all the nodes enables LEACH to achieve a longer lifetime than static clustering. LEACH is not as efficient as LEACH-C.

Lee et al [7] define an energy consumption model. It shows the impact the coverage aging process of a sensor network, i.e., how it degrades over time as some nodes become energy-depleted. To evaluate sensing coverage with heterogeneous deployments, we use total sensing coverage, which represents total information that can be extracted from all functioning sensors in a network area. Energy consumption model determines a device lifetime by considering application specific event characteristics, and network specific data extraction model and communication method. High-cost devices can function as a cluster-head or sink to collect and process the data from lowcost sensors, which can enhance the duration of network sensing operation.

Liang et al[8] proposes an Energy efficient method for data gathering to prolong network lifetime. The objective is to maximize the network lifetime without any knowledge of future query arrivals and generation rates. In other words, the objective is to maximize the number of data gathering queries answered until the first node in the network fails. The Algorithm MNL significantly outperforms all the other algorithms in terms of network lifetime delivered.

Basu et al[9] discusses about the data dissemination and gathering.. A majority of 
sensor networking applications involve data gathering and dissemination, hence energy efficient mechanisms of providing these services become critical. However, due to the broadcast nature of the wireless channel many nodes in the vicinity of a sender node overhear its packet transmissions even if those are not the intended recipients of these transmissions. This redundant reception results in unnecessary expenditure of battery energy of the recipients. Turning off neighboring radios during a certain point-topoint wireless transmission can mitigate this cost. To overcome this, Energy -Efficient data gathering and Dissemination algorithm is used.

\section{PRELIMINARIES OF PROPOSED ALGORITHM}

\subsection{Energy Consumption Model}

The energy consumption model determines the sensor lifetime. The energy calculation for a single cycle is done by using the following equation:

$$
\text { Ecycle }=E D+E S+E T+E R
$$

Where $E D, E S, E T$ and $E R$ represent the energy required for data processing, sensing, transmitting and receiving per cycle time, respectively. The quantity of energy spent for each operation depends on the network and the event model.

\subsection{Energy Maps}

The energy map, the component that contains information concerning the remaining available energy in all network areas, can be used to prolong the network lifetime.

\subsection{Qos Requirements}

The Qos requirements are based on energy, cost is used.

\subsection{Data Collection Mechanism}

Generally, sensor networks contain a large quantity of nodes that collect measurements before sending them to the applications. If all nodes forwarded their measurements, the volume of data received by the applications would increase exponentially, rendering data processing a tedious task. Data aggregation and data filtering are two methods that reduce the quantity of data received by Applications. The aim of those two methods is not only to minimize the energy consumption by decreasing the number of messages exchanged in the network but also to provide the applications with the needed data without needlessly overloading them with exorbitant quantities of messages. The aggregation data mechanism allows for the gathering of several measures into one record whose size is less than the extent of the initial records. However, the result semantics must not contradict the initial record semantics. Moreover, it must not lose the meanings of the initial records. The data filtering mechanism makes it possible to ignore measurements considered redundant or those irrelevant to the application needs. An example of a selective query is "SELECT the humidity readings FROM all sensors WHERE the temperature is above 40。 for DURATION of 2 hours EVERY 5 minutes". There are two main directions in query processing for optimizing the data collection process for selective queries: (1) preventing that a query is sent to nodes that do not fall into the scope of that query and, therefore, are not aware of the query and do not need to respond, and (2) minimizing the number of non-participating nodes in the collection path.

\subsection{A Dynamic Tabu Search Approach}

In order to facilitate the usage of tabu search for CBP, a new graph called Grow is defined. It is capable of determining feasible clusters. A feasible cluster consists of a set of nodes. Five steps should be conducted in order to adapt tabu search heuristics to solve a particular problem:1.Design an algorithm that returns an initial solution,2.Define moves that determine the neighbourhood $\mathrm{N}$ of a solution s,3.Determine the content and size of tabu lists,4.Define the 
aspiration criteria,5.Design intensification and diversification mechanisms.

\section{Initial solution:}

The goal is to find an appropriate initial solution for the problem, in order to get the best solution from tabu search iterations within a reasonable delay.the cost $\mathrm{c}_{\mathrm{a}}$ is found.

\section{The Neighborhood definition:}

It involves a move involving a regular node, a move involving an active node and a move involving a cluster head.The Cost $c_{b}$ is found.

\section{Increase Tabu lists:}

Our adaptation proposes two tabu lists: a reassignment list and a re-election list. The size of the tabu list is to be increased.when $\mathrm{C}_{\mathrm{a}}=\mathrm{C}_{\mathrm{b}}$ then the tabu list id increased and updated.

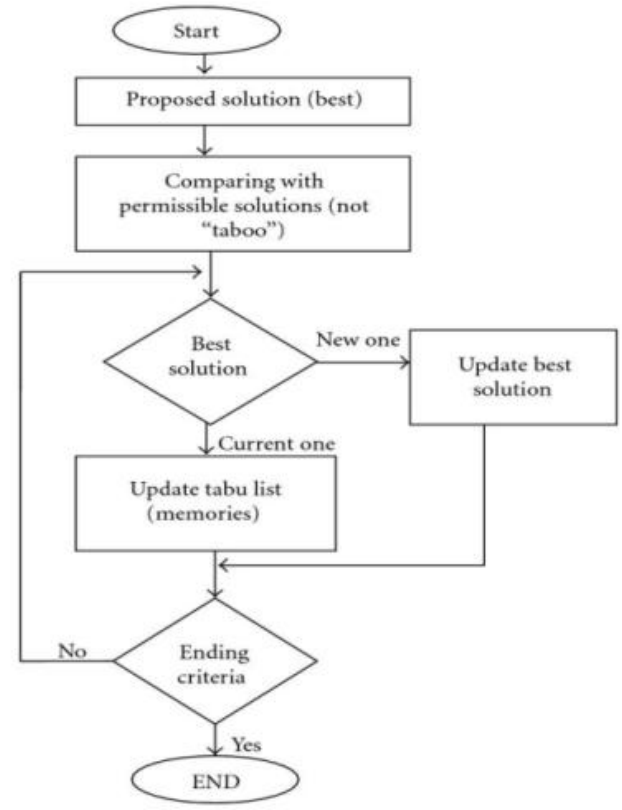

Figure 1 - Flow Diagram for modified Tabu Search for Clustering

\section{Aspiration criteria:}

Aspiration criterion, which consists of considering a move inventoried in the tabu list, which in turn, engenders a solution that is superior to the best solution found in the first place.

Design intensification and diversification mechanisms:

Diversification and Intensification are two mechanisms that make it possible to improve tabu search methods. They start by analysing the appropriate solutions. visited and obtain their common properties in order to be able to intensify the search in another neighborhood or to diversify the searches. The long and short term memory is used. The algorithm ends when one of the following 1. All possible moves are prohibited by the tabu lists;

2. The maximal number of iterations allowed has been reached;

3. The maximal number of iterations, where the best solution is not enhanced successively, has been reached.

\subsection{Mnl (Maximum_Network_Lifetime)}

Algorithm: The wireless sensor network $\mathrm{M}(\mathrm{N} ; \mathrm{A})$ is treated as a directed graph $\mathrm{G}(\mathrm{V} ; \mathrm{E})$, where the set of nodes $\mathrm{V}$ consisting of sensors and $(\mathrm{u} ; \mathrm{v}) € \mathrm{E}$ if and only if $\mathrm{u}$ and $\mathrm{v}$ are within the transmission ranges of each other. The basic idea is that, once a data gathering query arrives, a data gathering tree for the query is constructed using a greedy policy that maximizes the minimum residual energy among the nodes.

Specifically, the nodes are included into the tree one by one. Initially, only the sink node is included. Each time a node $\mathrm{v}$ is included into the tree, either the network lifetime derived from the current tree is at least as long as that without the inclusion of $\mathrm{v}$ to the tree or the amount of reduction of the network lifetime is minimized. In other words, a node $\mathrm{v}$ is chosen to be included into the tree if it leads to maximizing the minimum residual energy among the tree nodes including itself. 


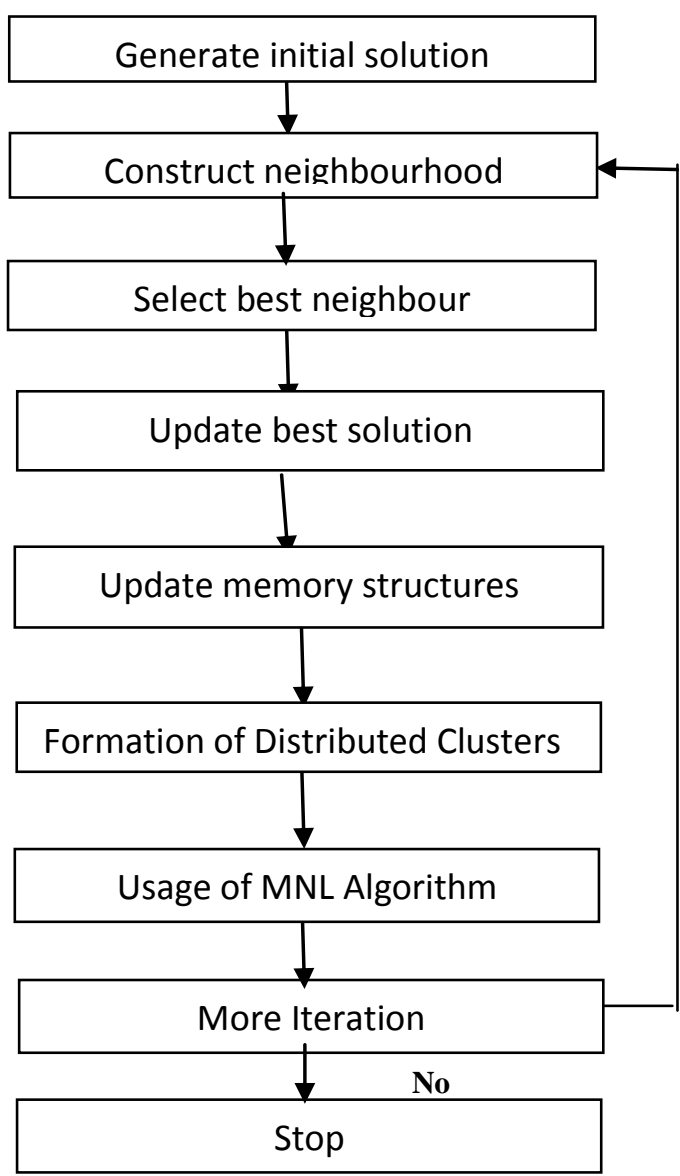

Figure 2-Tabu Search Algorithm and MNL algorithm for Distributed Clustering.

\section{Simulation Result and Discussion}

\subsection{Comparison between Centralized and Distributed approach}

The figure shows, the energy consumed to build the clusters by the centralized and distributed approaches using Dynamic tabu search approach. Results show that the distributed approach needs less energy consumption than the centralized approach and the gap between these energies becomes bigger when the network size increases.

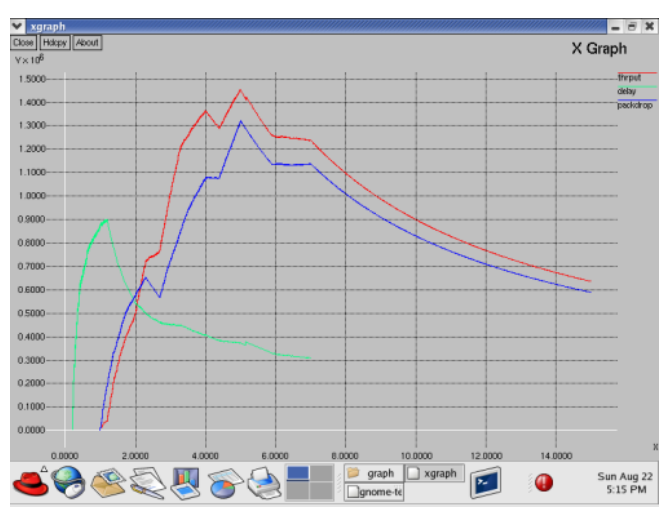

Figure 3 - Centralized Approach

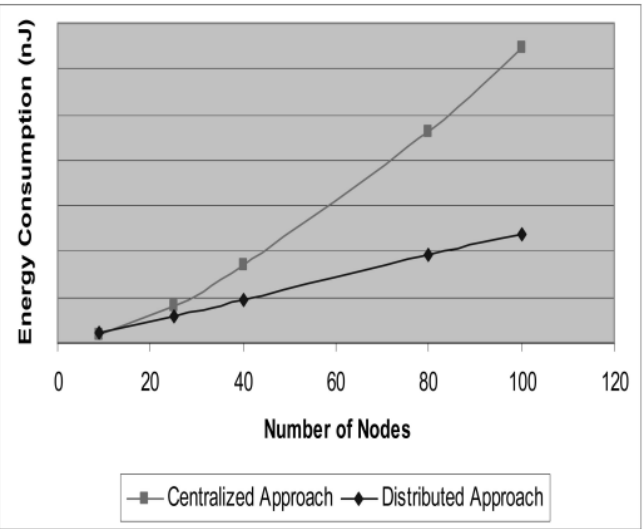

Figure 4- Comparison between Centralized and Distributed Approach

The reason behind this result is that the central node needs to generate a considerable number of messages in order to collect all the node information. The dynamic tabu search is good when compared to the normal tabu search in the memory storage.

\section{Conclusions}

This paper has presented a heuristic approach based on a energy efficient search to solve clustering problems where the numbers of 
clusters and cluster heads are unknown beforehand. The tabu search adaptation consists of defining three types of moves that allow reassigning nodes to clusters, selecting cluster heads, and removing existing clusters. Such moves use the largest size clique in a feasibility cluster graph, which facilitates the analysis of several solutions and makes it possible to compare them using a gain function. Performance of distributed approach with those of a centralized approach and we conclude that the central approach is less efficient than the distributed approach in the cluster building phase.

\section{References}

[1]. O. Moussaoui, A. Ksentini, M. Naimi, and M. Gueroui, "A Novel Clustering Algorithm for Efficient Energy Saving in Wireless Sensor Networks," Proc. Seventh Int'l Symp. Computer Networks (ISCN '06), pp. 66-72, 2006.

[2].S. Raghuwanshi and A. Mishra, "A SelfAdaptive Clustering Based Algorithm for Increased Energy-Efficiency and Scalability in Wireless Sensor Networks," Proc. IEEE 58th Vehicular Technology Conf. (VTC '03), vol. 5, pp. 2921-2925, 2003.

[3]. O. Younis and S. Fahmy, "Distributed Clustering in Ad-Hoc Sensor Networks: A Hybrid, Energy-Efficient Approach," Proc. IEEE INFOCOM, pp. 629-640, 2004.

[4] Suchismita Chinara , Santanu Kumar Rath "Energy Efficient Mobility Adaptive Distributed Clustering Algorithm for Mobile Ad Hoc Network," Proc ADCOM 2008,pp 265-272,2008

[5].A. El Rhazi and S. Pierre, "A Data Collection Algorithm Using Energy Maps in Sensor Networks," Proc. Third IEEE Int'l Conf. Wireless and Mobile Computing, Networking, and Comm. (WiMob '07), 2007.
[6]. W. Heinzelman, A. Chandrakasan, and H. Balakrishnan, "An Application Specific Protocol Architecture for Wireless Microsensor Networks," IEEE Trans. Wireless Comm., vol. 1, no. 4, pp. 660-670, Oct. 2002.

[7]. J.J. Lee, B. Krishnamachari, and C.C.J. Kuo, "Impact of Heterogeneous Deployment on Lifetime Sensing Coverage in Sensor Networks," Proc. IEEE Sensor and Ad Hoc Comm. and Networks Conf. (SECON '04), pp. 367-376, 2004.

[8].W. Liang and Y. Liu, "Online Data Gathering for Maximizing Network Lifetime in Sensor Networks," IEEE Trans. Mobile Computing, vol. 6, no. 1, pp. 2-11, Jan. 2007

.[9]. P. Basu and J. Redi, "Effect of Overhearing Transmissions on Energy Efficiency in Dense Sensor Networks," Proc. Third Int'l Symp. Information Processing in Sensor Networks (IPSN '04), pp. 196- 204, Apr. 2004. 